\title{
Discapacidad y emprendimiento. Un estudio cualitativo desde una perspectiva competencial
}

\author{
Ángel José Olaz Capitán \\ Universidad de Murcia \\ olazcapi@um.es \\ Pilar Ortiz García \\ Universidad de Murcia \\ portizg@um.es
}

Resumen: Este trabajo pretende realizar una aproximación al fenómeno del emprendimiento en aquellas personas con discapacidad física y sensorial a través de un estudio competencial. En concreto, el estudio identifica las dimensiones y aspectos competenciales que propician y/o limitan la actividad emprendedora de este colectivo. Para ello, se ha utilizado la técnica de grupo nominal. Este artículo explora el perfil de la persona discapacitada en España para situar el papel concedido al emprendimiento - desde una perspectiva sociológica-y abordarlo de un modo más preciso en personas que presentan algún tipo de discapacidad física y sensorial.

Palabras clave: discapacidad; emprendimiento

\begin{abstract}
The present study examines the phenomenon of entrepreneurship among people with physical and sensory disabilities. Specifically, the study identifies the skills that promote and/or limit the entrepreneurial activity of people who belong to this group. To do so, the study has used the nominal group technique. The article explores the profiles of disabled people in Spain to determine the sociological role of entrepreneurship among them and examines it in greater detail in relation to people who have some form of physical and sensory disability.
\end{abstract}

Keywords: disability; entrepreneurship 


\section{Planteamiento del problema}

Este trabajo pretende realizar una aproximación al fenómeno del emprendimiento en aquellas personas con discapacidad física y sensorial a través de un estudio competencial.

En concreto, el estudio identifica las dimensiones y aspectos competenciales que propician y/o limitan la actividad emprendedora de este colectivo. Para ello, se ha utilizado la técnica de grupo nominal (en adelante, TGN) ${ }^{1}$.

Este artículo explora el perfil de la persona discapacitada en España para situar el papel concedido al emprendimiento - desde una perspectiva sociológica- $y$ abordarlo de un modo más preciso en personas que presentan algún tipo de discapacidad física y sensorial.

Tras el estudio de estos aspectos, se hará alusión a diferentes perspectivas desde la que enfocar el término competencia antes de realizar la justificación metodológica, enfatizando en el papel conferido en este estudio a la TGN empleada en varios colectivos consultados (técnicos de la Administración, directivos y técnicos de asociaciones de personas discapacitadas) - y su conexión con los aspectos competenciales, necesarios para desarrollar el proceso de emprendimiento, a través del test emotional competence intelligence (ECI) como vehículo para la identificación de competencias en el proceso de emprendimiento en personas con discapacidad-.

Este trabajo finaliza considerando las principales, así como un conjunto de recomendaciones que revertirían y pondrían en valor el emprendimiento de las personas con discapacidad en clave competencial

\section{El papel del emprendimiento desde una perspectiva sociológica. Una aproximación al tema objeto de estudio}

El estudio sobre el emprendimiento y los factores que lo desencadenan ha cobrado en la actualidad una especial relevancia. Uno de los motivos ha sido la difícil situación económica que atraviesan las economías poscrisis y muy especialmente la española. La alta tasa de desempleo que ha dejado el paso de la crisis ha provocado la atención de políticos y académicos en las estrategias que fomentan el autoempleo y/o el emprendimiento. Unida a este motivo está la emergencia de

1 El estudio se desarrolla al amparo del Proyecto Discapacidad y Emprendimiento. Análisis Competencial (CSO2016 - 75818 - R), convocatoria para el año 2016, correspondiente al Programa Estatal de Fomento de la Investigación Científica y Técnica de Excelencia, Subprograma Estatal de Generación de Conocimiento, en el marco del Plan Estatal de Investigación Científica y Técnica y de Innovación 2013-2016, financiado por el Ministerio de Economía y Competitividad del Gobierno de España. 
un discurso que eleva el emprendimiento a la categoría de milagro o, más modestamente, receta eficaz frente al paro.

Ello no obsta para que, desde una fundamentada perspectiva crítica, se alcen algunas voces que identifican estas iniciativas emprendedoras con una forma de autoempleo (Renko et alii, 2016) en la que prima la «necesidad» sobre la «oportunidad» a la hora de iniciar una actividad empresarial (Santos, 2014; Muñoz y Santos, 2016; Suárez, 2017).

Sin entrar en debate sobre la diferencia terminológica entre los conceptos de emprendimiento y/o autoempleo, es cierto que la iniciativa empresarial resulta uno de los elementos que se manejan como medida para impulsar el empleo. El tener que abrir nuevas vías en el mercado laboral, de tomar iniciativas creativas e innovadoras que posibiliten puestos de trabajo, se ha convertido en una necesidad, por lo que han proliferado las iniciativas encaminadas a este fin.

En paralelo, han sido abundantes los estudios que tienen como objeto conocer el perfil del emprendedor (Schumpeter, 1984; Renau, 1994; Benavides et alii, 2004); los contextos y factores sociales que propician el emprendimiento; el tipo de iniciativas y las motivaciones que las promueven (Brockhaus, 1980; Bonnett y Furnham, 1991; Cromie, 2000; Markman y Baron, 2003; Sánchez, 2003; Jung et alii, 2001; Moriano et alii, 2006), y, en definitiva, las variables que explican el fenómeno emprendedor (Turró et alii, 2014). Esta literatura pone de manifiesto la necesidad de abrir nuevas líneas de investigación interdisciplinares, entre las que no puede faltar la aportación de la sociología. Desde esta disciplina se entiende la conducta humana en general $-y$ la del emprendedor en particular - como el resultado de conjugar aspectos del entorno social y características personales (Ortiz y Millán, 2010).

Desde un punto de vista sociológico, las condiciones laborales, la necesidad de mejora en el puesto de trabajo y la obtención de un reconocimiento social son factores que promueven la acción de emprender. Así lo recogen algunos de los estudios realizados sobre el tema por Shapero y Sokol (1982) o Evans y Lighton (1989). También autores como Moriano (2005) inciden en los factores del entorno social — tales como los antecedentes familiares, la experiencia o aprendizaje previo-, el sistema de valores, y orientaciones societales que fomentan o inhiben el surgimiento de emprendedores. Para explicar de forma gráfica este hecho, el autor se refiere a nichos ecológicos de emprendedores, o, si se prefiere, marcos espacio-temporales donde es previsible que surjan emprendedores (Moriano, 2005: 15).

En la misma línea Ajzen $(1987 ; 1988)$, tratando de buscar un paradigma que explique la intención y la actitud emprendedora, ya había analizado el tema en su teoría de la acción planificada. Se trata de un modelo teórico que estudia la 
conducta emprendedora desde una perspectiva en la que confluyen tanto aspectos personales como sociales, a la vez que insiste en la presión que ejercen las personas que nos rodean sobre el desarrollo de la conducta. La base de esta teoría es el protagonismo que le confiere a la presión del entorno familiar, educativo y laboral, en definitiva, social, en el surgimiento de la conducta emprendedora. Desde el punto de vista del autor, los grupos de referencia y/o de pertenencia actúan como modelos que explican la intención emprendedora a partir de la interacción entre aspectos personales y sociales. Se trata, por tanto, de una teoría que proporciona un sólido marco teórico para explicar y predecir la intención de crear una empresa o del autoempleo.

Incidiendo en los mismos aspectos sociales, Ortiz (2016), en una investigación sobre emprendimiento y jóvenes, analiza la influencia del capital social colectivo en el desarrollo de la conducta emprendedora, llegando a la conclusión de que las redes sociales guardan una relación positiva con la actitud de los jóvenes ante el emprendimiento, especialmente las que tienen que ver con la clase social. También los estudios de Olaz y Ortiz (2016a; 2016b) y Ortiz y Olaz (2016a; 2016b) sobre mujer y emprendimiento hallan conexiones entre las iniciativas emprendedoras de las mujeres y ciertas competencias individuales y sociales, como las relacionadas con el autoconocimiento, la autoevaluación, la conciencia emocional, la capacidad para gestionar los conflictos o el trabajo en equipo.

\section{Fundamentación teórica del emprendimiento en las personas con discapacidad}

A pesar de los avances sociales de las últimas décadas, la integración de las personas con discapacidad sigue siendo una cuestión pendiente. Mejorar la empleabilidad de este colectivo es un reto social de primera magnitud. Entre los objetivos enunciados por programas tales como Europa 2020, de la Comisión Europea, figura la consecución de sociedades inclusivas. En este sentido, una de las primeras medidas es conocer los distintos problemas a los que se enfrentan colectivos en riesgo de exclusión social para diseñar políticas que la combatan en cualquiera de sus formas. Las personas con discapacidad son uno de los colectivos más expuestos a situaciones de vulnerabilidad.

Dada la amplitud del concepto y la variada casuística que recoge, resulta oportuno aclarar lo que se entenderá por discapacidad, concepto establecido por la Clasificación Internacional del Funcionamiento, de la Discapacidad y de la Salud, que define la discapacidad como un término genérico que engloba deficiencias, limitaciones de actividad y restricciones para la participación de la persona en actividades consideradas normales para cualquier individuo de sus 
características. La discapacidad denota los aspectos negativos de la interacción entre personas con un problema de salud (como parálisis cerebral, síndrome de Down o depresión) y factores personales y ambientales (como actitudes negativas, transporte y edificios públicos inaccesibles, y falta de apoyo social). Así se pone de manifiesto en el Informe Mundial de Discapacidad de la Organización Mundial de la Salud (OMS, 2001).

Según este informe, el colectivo de personas discapacitadas supone más de mil millones en el mundo (un 15\% de la población mundial según las estimaciones de 2010 recogidas en la Encuesta Mundial de Salud). En España, la información que se recoge en la base estatal del Instituto de Mayores y Servicios Sociales (IMSERSO) es la relativa al histórico de las personas que han solicitado el reconocimiento de la situación de discapacidad, así como el resultado de las valoraciones efectuadas por los equipos de evaluación de las distintas comunidades autónomas, incluidas las ciudades autónomas de Ceuta y Melilla (con información actualizada a 31 de diciembre de 2015). Del resultado de este proceso de valoración se obtiene un total de 2.998.639 personas con discapacidad, es decir, con un grado de discapacidad reconocido igual o mayor al 33\%, de acuerdo con lo que establece el artículo 4.2 del Real Decreto Legislativo 1/2013, de 29 de noviembre, por el que se aprueba el Texto Refundido de la Ley General de derechos de las Personas con Discapacidad y de su Inclusión Social.

Atendiendo a las tipologías, las discapacidades se distribuyen de la siguiente forma: osteoarticular (28,05\%), enfermedad crónica (18,01\%), mental $(16,06 \%)$, neuromuscular $(10,13 \%)$, intelectual $(8,96 \%)$, visual $(7,00 \%)$, auditiva $(5,79 \%)$, mixta $(2,67 \%)$, expresiva $(0,50 \%)$, y un porcentaje residual correspondiente a las categorías «otras» $y$ «no consta».

Estas cifras se mantienen al alza debido a varios factores. Por una parte, la evolución demográfica en la que se observa un proceso cada vez más intenso del envejecimiento. Por otro lado, los modos de vida, que han producido un incremento global de enfermedades crónicas (tales como problemas cardiovasculares, trastornos mentales, diabetes, etc.), sin olvidar otros derivados de factores ambientales y también sociales.

Estos datos sitúan a este colectivo en una situación de vulnerabilidad importante, dada la mayor probabilidad de caer en situaciones de exclusión social. Una de las formas más importantes de inclusión es el trabajo, la consecución de un empleo y su desempeño. El trabajo constituye una de las formas más eficaces de proporcionar cohesión social, a la vez que independencia, seguridad económica y sentido de pertenencia social para individuo.

La información sobre inserción laboral de personas con discapacidad aparece recogida en diversas fuentes. En España se han realizado tres macroencuestas 
por parte del Instituto Nacional de Estadística (INE), en 1986, 1999 y 2008: la Encuesta sobre Discapacidades, Deficiencias y Minusvalías (EDDM 1986), la Encuesta sobre Discapacidades, Deficiencias y Estado de Salud (EDDS 1999) y la última Encuesta de Discapacidad, Autonomía personal y situaciones de Dependencia (EDAD 2008). No obstante, el contenido de estas encuestas, siendo esencial para el conocimiento del colectivo, contiene datos muy básicos sobre actividad económica y en ningún caso sobre intención o materialización de algún tipo de actividad emprendedora. Dicha información sí aparece en la encuesta El Empleo de las Personas con Discapacidad, del INE, aunque con un limitado nivel de desagregación y detalle.

Este vacío parcial se pone de manifiesto en la producción científica sobre el tema, que es tratado de forma transversal en diferentes disciplinas (sociología, psicología, medicina, etc.), sin que en ellas se acabe consolidando una especialidad propiamente reconocida. La sociología da cuenta de una reseñable producción científica desde finales de los años ochenta. En España cabe mencionar los estudios de Jiménez Lara (1991; 1992; 1998a; 1998b; 2001; 2002; 2005; 2007), Casado (1991), Jiménez y Huete (2002a; 2002b; 2003), Rodríguez Cabrero (2002), Rodríguez y Pérez (2004), De Lorenzo (2003), Zarco y García de la Cruz (2004), Pérez Bueno (2004), Fernández-Cid (2005), Díaz Casanova (1985), Bascones y Quezada (2006), Romañach (2002), Romañach y Palacios (2007), y los de Vázquez Ferreira (2005, 2007a; 2007b; 2008; 2010; 2011), entre otros.

No obstante, esta producción queda lejos de la generada sobre este tema en la tradición investigadora anglosajona. Desde dicha tradición se parte de la hipótesis que lleva a concebir la discapacidad como una construcción social que estigmatiza a las personas con algún tipo de limitación de sus capacidades físicas o psíquicas. Esta idea lleva a que las políticas activas dirigidas a la inclusión de personas con discapacidad se introduzcan en la agenda social, ya que se trata de una cuestión que afecta a todos.

La discapacidad es un fenómeno multidimensional que afecta a la persona desde una perspectiva biológica, psicológica y social. De todas ellas, la dimensión social ha sido una de las menos estudiadas (Vázquez Ferreira, 2008). Sin embargo, la determinación de los factores sociales sobre los biológicos y psicológicos es clara, de ahí la oportunidad de estudios que propicien actuaciones encaminadas a la normalización e integración plena de las personas con discapacidad en los entornos en los que interactúan.

El entorno laboral resulta clave para conseguir la normalización a la que se ha hecho referencia y, en particular, las experiencias emprendedoras. Conocer las posibilidades de las personas con discapacidad a partir del diagnóstico de compe- 
tencias que las hacen especiales para acometer empresas de valor social, ecológico y humano se convierte en un objetivo fundamental de cualquier sociedad que aspire a alcanzar la calificación de «inclusiva».

El observatorio Global Entrepreneurship Monitor (GEM) constituye una de las principales bases de datos internacional sobre la actividad emprendedora. Sin embargo, no incluye un apartado específico que permita el seguimiento de dicha acción en personas con discapacidad.

De todo ello se desprende que, si bien se está avanzando en la recopilación de información y se dispone de fuentes estadísticas y estudios sobre el tema de discapacidad y emprendimiento, no resultan suficientes $y$, sobre todo, no son lo suficientemente específicas sobre esta cuestión.

\section{Diseño metodológico}

Los aspectos metodológicos de este trabajo motivan el enfatizar en las dos herramientas empleadas en su desarrollo: la técnica de grupo nominal y el test emotional competence intelligence (se denominará ECI).

Justifica la utilización de ambos instrumentos la estrategia metodológica adoptada, en la que se pretendió llegar a una definición de las dimensiones significativas para el análisis del objeto de estudio y su concreción en aspectos competenciales del emprendimiento en el colectivo estudiado. Siguiendo esta estrategia, se definieron, en primer lugar, las dimensiones o ámbitos de estudio del emprendimiento en personas con discapacidad a través de la realización de cuatro TT. GG. NN. (directivos y técnicos de asociaciones, técnicos de la Administración pública y personas discapacitadas), y, sobre esa base, se sometieron a discusión cuáles eran las competencias clave moderadoras del emprendimiento a través del cuestionario ECI.

Como resultado de esta triangulación metodológica se pudieron detectar qué competencias incidían en el emprendimiento de las personas con discapacidad, según ámbitos de estudio y colectivos representados.

Las siguientes líneas explicarán las características de ambos elementos y su imbricación metodológica.

Sobre el modelo original descrito para la técnica de Van de Ven y Delbecq, varios son los elementos que han sido variados en este proyecto de investigación, sin por ello querer faltar a sus objetivos principales, como son identificar y enriquecer la comprensión acerca del problema a través de un discurso susceptible de cuantificación; establecer un conjunto de hipótesis relacionadas con el significado y efectos del problema investigado y centrar la atención en las principales áreas o escenarios de investigación (Van de Ven y Delbecq, 1972:338). 
Tampoco se ha querido renunciar a sus objetivos, esto es, identificar las dimensiones críticas de un problema por medio de un proceso grupal no amenazante y despersonalizado; aclarar el significado de los puntos críticos, tanto para investigadores como para los participantes, a través de la acción discursiva; explorar objetiva y subjetivamente las dimensiones que envuelven al problema objeto de estudio; identificar los pesos explicativos a través de procedimientos de votación (en este caso debería hablarse mejor de establecimiento de una prelación), y contribuir al desarrollo de hipótesis y la formulación de la redacción de preguntas orientadas a la investigación y entrevistas en posteriores trabajos de campo (Van de Ven y Delbecq, 1972: 341).

Bajo estos supuestos, Gutiérrez (2011: 117), en referencia a las técnicas de investigación cualitativas, tanto por su inconsistencia técnica como la aparente simpleza de su aplicación práctica, sugiere la utilización de prácticas artesanales no totalmente formalizadas $y / o$ estandarizadas, extremadamente singulares $y$ virtuosas, bien sea en referencia a los procesos desarrollados como a los productos alcanzados, que al final están muy condicionadas a la figura del investigador.

Estas cuestiones explicarían las adaptaciones metodológicas que se realizan, por ejemplo, a nivel muestral, buscando una mejora en la gestión del tiempo de sesión (Dobbie et alii, 2004: 402-406) y de tantos otros investigadores que, quizá por no despertar críticas, silencien sus adaptaciones procedimentales.

Estas cuestiones, unidas a la reflexión de Ruiz (2012: 160), que evitaría hablar de técnicas cualitativas y más de prácticas cualitativas, dado el carácter abierto y flexible de los procedimientos por los que se aborda la investigación social cualitativa, ayudarían a comprender la adaptación de la técnica de grupo nominal a nuestro estudio. En el intento por establecer un diálogo entre el modelo establecido por Delbecq y Van de Ven (1971) y Delbecq, Van de Ven y Gustafson (1975) y la adaptación del modelo realizado, el cuadro 1 recoge la sucesión de etapas que jalonan ambos esquemas.

Cuadro 1. Fases comparadas de los modelos

\begin{tabular}{|c|c|c|c|}
\hline FASE & $\begin{array}{c}\text { ADAPTACIÓN } \\
\text { METODOLÓGICA }\end{array}$ & FASE & $\begin{array}{c}\text { TGN } \\
\text { CLÁSICA }\end{array}$ \\
\hline Fase 1 & Introducción. & Fase 1 & Introducción. \\
\hline Fase 2 & Generación silenciosa de ideas. & Fase 2 & Generación silenciosa de ideas. \\
\hline Fase 3 & Agrupación de personas. & Fase 3 & Listado Round-Robin. \\
\hline Fase 4 & Discusión en grupo. & Fase 4 & Discusión en grupo. \\
\hline Fase 5 & Selección y jerarquización tarjetas. & & \\
\hline Fase 6 & Colocación de las tarjetas. & & \\
\hline Fase 7 & Pausa o coffee break. & Fase 5 & Pausa o coffee break. \\
\hline
\end{tabular}




\begin{tabular}{|c|c|c|c|}
\hline & & Fase 6 & $\begin{array}{l}\text { Listado priorizado de elementos } \\
\text { «problemáticos». }\end{array}$ \\
\hline & & Fase 7 & Discusión y votación. \\
\hline & & Fase 8 & $\begin{array}{l}\text { Reactualización del ranking de } \\
\text { prioridades. }\end{array}$ \\
\hline & & Fase 9 & Conclusión. \\
\hline Fase 8 & Discusión seriada de las ideas plasmadas. & & \\
\hline Fase 9 & Cuantificación del peso de los escenarios. & & \\
\hline Fase 10 & Desarrollo de un plan de acción. & & \\
\hline Fase 11 & $\begin{array}{l}\text { Asociación de competencias } \\
\text { a planes de acción. }\end{array}$ & & \\
\hline Fase 12 & Constitución de los EE AA TT. & & \\
\hline
\end{tabular}

Fuente: elaboración propia.

El modelo empleado en la investigación supone una adaptación de esta herramienta al fenómeno del emprendimiento. El modelo identifica cuatro grandes dimensiones (autoconocimiento personal, autogestión, conciencia social y gestión de relaciones) que contienen un total de 18 aspectos competenciales; dichos aspectos son captados a partir de 18 cuestiones, cada una de las cuales se traslada a una escala Likert de cuatro niveles de respuesta (ver cuadro 2).

\section{Cuadro 2, Modelo ECI}

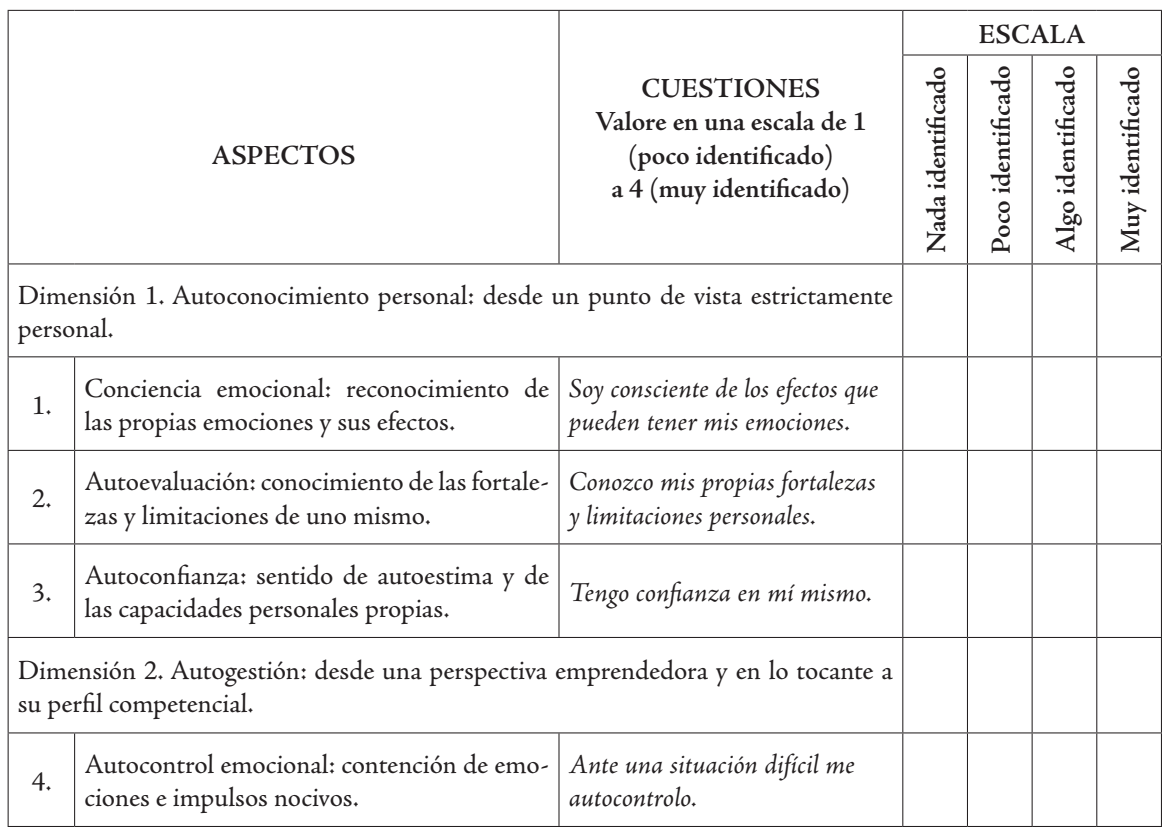




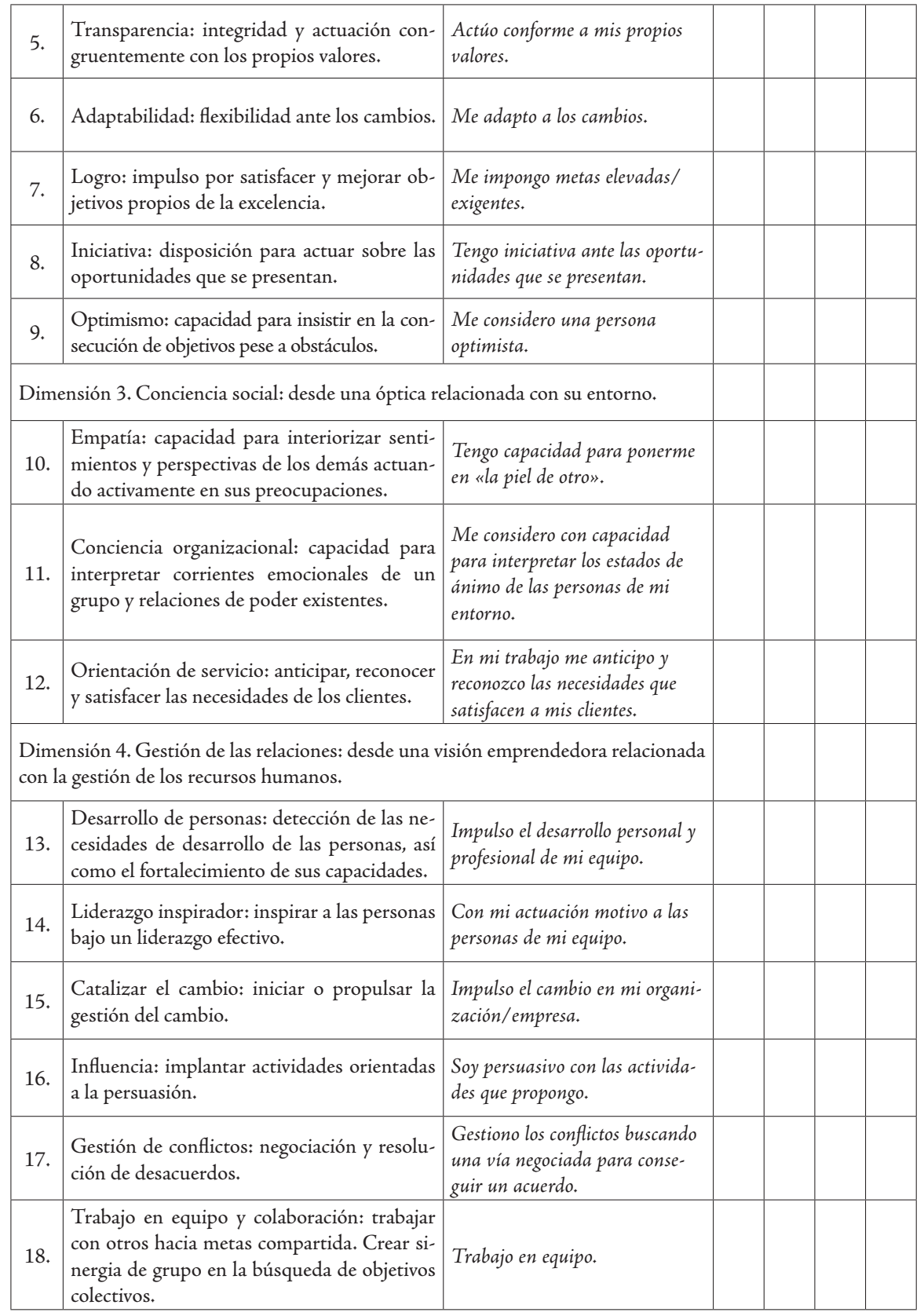

Fuente: elaboración propia a partir de Hay Group, McClelland Center for Research and Innovation. Prepared by Steven B. Wolff, DBA. Updated November 2005 Emotional Competence Inventory (ECI) Technical Manual. 
En síntesis, el trabajo consistió en pedir, y debatir después, a los asistentes a las diferentes TT. GG. NN. que identificaran qué competencias de las 18 existentes favorecerían el emprendimiento en cada uno de los escenarios definidos con anterioridad.

\section{Resultados y discusión}

Un somero análisis de las dimensiones señaladas en las diferentes TT. GG. NN. (ver cuadro 3) indica una importante coincidencia - con independencia del colectivo participante (directivos y técnicos de asociaciones; técnicos de la Administración pública y personas discapacitadas) - acerca de bajo qué contextos se identifican aspectos que contribuyen a dificultar el emprendimiento en personas con discapacidad.

\section{Cuadro 3. Comparativa de escenarios por colectivos intervinientes en las TT. GG. NN.}

\begin{tabular}{|l|c|c|c|c|}
\hline \multicolumn{1}{|c|}{ ESCENARIOS } & $\begin{array}{c}\text { TGN } \\
\text { Personas } \\
\text { discapacitadas }\end{array}$ & $\begin{array}{c}\text { TGN } \\
\text { Técnicos } \\
\text { AA. PP. }\end{array}$ & $\begin{array}{c}\text { TGN } \\
\text { Asociaciones } \\
\text { técnicos }\end{array}$ & $\begin{array}{c}\text { TGN } \\
\text { Asociaciones } \\
\text { directivos }\end{array}$ \\
\hline Aspectos personales-psicológicos & $24,0 \%$ & $31,6 \%$ & $20,0 \%$ & $38,2 \%$ \\
\hline Aspectos socioculturales & $17,0 \%$ & $18,4 \%$ & $34,0 \%$ & $15,1 \%$ \\
\hline Aspectos institucionales-legales & $25,0 \%$ & $27,0 \%$ & $13,0 \%$ & $13,8 \%$ \\
\hline Aspectos educativo-formativos & $10,0 \%$ & $15,1 \%$ & $16,0 \%$ & $23,7 \%$ \\
\hline Aspectos socioeconómicos & $24,0 \%$ & $1,3 \%$ & $17,0 \%$ & $0,0 \%$ \\
\hline Aspectos familiares & $0,0 \%$ & $0,0 \%$ & $0,0 \%$ & $9,2 \%$ \\
\hline Aspectos físicos-accesibilidad & $0,0 \%$ & $6,6 \%$ & $0,0 \%$ & $0,0 \%$ \\
\hline Total & $100,0 \%$ & $100,0 \%$ & $100,0 \%$ & $100,0 \%$ \\
\hline
\end{tabular}

Fuente: elaboración propia.

En este sentido y en su conjunto, los aspectos donde se encuentra mayor número de limitaciones se localizan en la esfera del terreno personal y psicológico del individuo, seguida del espacio social, donde los elementos culturales son especialmente significativos. También el espacio institucional y el ámbito legal presentan elementos inhibidores del emprendimiento junto con los educativos y formativos. El terreno socioeconómico y el escenario familiar, siendo importantes, no se consideran por parte de todos los colectivos como ámbitos donde pueden localizarse elementos que perjudiquen el emprendimiento. 
Profundizando un poco más en detalle en la TGN realizada a personas con discapacidad respecto a los factores limitadores del emprendimiento, destacan los institucionales-legales (25\%), que representan el mayor peso explicativo en el conjunto de aspectos considerados. Los integrantes de la TGN destacan las limitaciones que suponen para el emprendimiento cuestiones tales como la falta de vías de financiación adecuadas a las necesidades particulares de colectivos como el que nos ocupa. Junto con estos aspectos, también se denuncia la falta de oferta formativa para el emprendimiento. Otro grupo de factores serían las trabas burocráticas que acompañan a los procedimientos económicos y que, para estas personas, pueden suponer una dificultad adicional. Por último, se demanda mayor sensibilidad para la eliminación de barreras físicas, así como la dotación de medios humanos y técnicos especialmente pensados para el colectivo, como pueden ser traductores.

Los aspectos personales-psicológicos, junto con los socioeconómicos, ocupan un segundo lugar en la consideración de los participantes en esta TGN, con un peso del 24\%. En cuanto a los primeros, resultan relevantes las cuestiones derivadas de la propia discapacidad, que ralentizan aspectos como el aprendizaje. También destacan las derivadas de la falta de empatía observada en la sociedad respecto a las dificultades con las que se tienen que enfrentar los discapacitados en su día a día. Síntomas como la desmotivación, la baja autoestima y la poca confianza en sí mismos son el resultado de la falta de comprensión percibida en el entorno.

En cuanto a los aspectos socioeconómicos (24\%), si bien no resultan específicos de las personas con discapacidad, sí tienen algunas particularidades que, en el caso de este colectivo, vienen a dificultar un posible emprendimiento o su desarrollo, en caso de estar iniciado. Destacan cuestiones tales como la falta de inversión económica en apoyo técnico y humano para discapacitados, falta de inversión en aspectos directamente relacionados con las cuestiones empresariales y escasos o nulos incentivos fiscales.

Seguidos de los anteriores, y con un peso explicativo en la limitación al emprendimiento del 17\%, se han destacado los aspectos socioculturales. El común denominador a los límites señalados en este escenario es la falta de comprensión social sobre la discapacidad. Bajo esta premisa, los participantes relacionan cuestiones tan relevantes como las barreras en la comunicación social, la desconfianza que perciben en el resto de personas, y la invisibilidad de la discapacidad, y, con ella, de las dificultades que lleva aparejada cualquier actividad que afrontan. A estos aspectos se unen otros que, aun siendo transversales, como la edad, en este caso constituyen dificultades adicionales en la inserción profesional y en el emprendimiento en particular. 
Por último, en cuarto lugar, los participantes en esta TGN han identificado los aspectos educativo-formativos como elementos potencialmente limitadores del emprendimiento, con un peso explicativo significativamente menor que los anteriores (10\%). Entre las cuestiones mencionadas en este escenario, si bien existen elementos limitantes comunes para cualquier persona - discapacitada o no-, como son la falta de conocimientos de marketing y otros específicos de la gestión empresarial, se enuncian otros directamente relacionados con los límites percibidos por este colectivo. Son cuestiones tales como una ausencia de formación específica para emprendedores discapacitados u otros como la falta de recursos humanos de apoyo. Es el caso del colectivo con discapacidad auditiva, usuario de la lengua de signos, que de forma reiterada denuncia la falta de intérpretes de esta forma de comunicación.

Los hallazgos descritos relacionados con las dimensiones que, a juicio del colectivo objeto de estudio, limitan o impiden el emprendimiento se ven corroborados por las investigaciones sobre el tema. En este sentido, el resultado que apunta a la importancia de los factores institucionales, junto con los puramente personales, en la decisión de emprender, confirma los estudios de Ajzen (1987; 1988) sobre el tema. Desde esta perspectiva, los factores sociales ejercen una influencia significativa sobre la actividad emprendedora, máxime si se tiene en cuenta que se trata de un colectivo sobre el que planean prejuicios de carácter social que tienen su reflejo en la actitud, un factor de naturaleza psicológica.

De la misma forma, la incidencia de los sujetos en la importancia de la dimensión socioeconómica confirma los hallazgos de investigaciones de Moriano (2005) y Moriano et alii (2006) que inciden en la importancia de la creación de marcos espacio-temporales en los que se generen oportunidades emprendedoras.

Aunque de menor relevancia en los resultados analizados, también los factores educativos y de capital social resultan relevantes, en sintonía con la investigación de autores como Ortiz (2016) sobre el tema.

En términos competenciales (ver cuadro 4) y, a partir de los resultados obtenidos, se desprende la existencia de un alto nivel de correspondencia entre las competencias de las cuatro dimensiones y la solución a las limitaciones planteadas a la hora de emprender. En concreto, las competencias correspondientes a la dimensión 2, «autogestión», cubren todos los aspectos identificados en los cinco escenarios. Dentro de esta dimensión, adquieren especial relevancia, por su peso explicativo, las competencias siguientes: «optimismo» $(48,5 \%)$; «transparencia» (40\%) e «iniciativa» (40\%). El hecho de cubrir todos los escenarios, a la vez que contener competencias con un mayor peso en la aportación de soluciones a los problemas planteados en dichos escenarios, da cuenta de la importancia de esta dimensión. 
Cuadro 4. Distribución de competencias por escenarios para el colectivo de personas con discapacidad

\begin{tabular}{|c|c|c|c|c|c|c|c|}
\hline \multirow{2}{*}{\multicolumn{2}{|c|}{ Dimensión }} & \multicolumn{5}{|c|}{ Escenario } & \multirow[b]{2}{*}{ 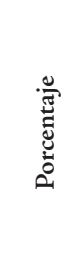 } \\
\hline & & 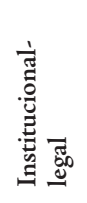 & 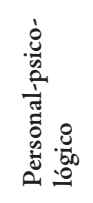 & 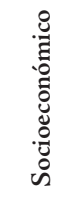 & 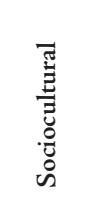 & 崫运 & \\
\hline \multicolumn{8}{|c|}{ Dimensión 1. Autoconocimiento personal: desde un punto de vista estrictamente personal. } \\
\hline 1 & \begin{tabular}{|l|} 
Conciencia emocional: reconocimiento \\
de las propias emociones y sus efectos.
\end{tabular} & $28,6 \%$ & $100,0 \%$ & $0,0 \%$ & $57,1 \%$ & $14,3 \%$ & $40,0 \%$ \\
\hline 2 & $\begin{array}{l}\text { Autoevaluación: conocimiento de las } \\
\text { fortalezas y limitaciones de uno mismo. }\end{array}$ & $0,0 \%$ & $85,7 \%$ & $14,3 \%$ & $14,3 \%$ & $42,8 \%$ & $31,4 \%$ \\
\hline 3 & $\begin{array}{l}\text { Autoconfianza: sentido de autoestima y } \\
\text { de las capacidades personales propias. }\end{array}$ & $0,0 \%$ & $85,7 \%$ & $42,8 \%$ & $57,1 \%$ & $14,3 \%$ & $40,0 \%$ \\
\hline \multicolumn{8}{|c|}{ Dimensión 2. Autogestión: desde una perspectiva emprendedora y en lo tocante a su perfil competencial. } \\
\hline 4 & $\begin{array}{l}\text { Autocontrol emocional: contención de } \\
\text { emociones e impulsos nocivos. }\end{array}$ & $14,3 \%$ & $71,4 \%$ & $14,3 \%$ & $28,6 \%$ & $42,8 \%$ & $34,3 \%$ \\
\hline 5 & $\begin{array}{l}\text { Transparencia: integridad y actuación } \\
\text { congruentemente con los propios va- } \\
\text { lores. }\end{array}$ & $14,3 \%$ & $100,0 \%$ & $14,3 \%$ & $28,6 \%$ & $42,8 \%$ & $40,0 \%$ \\
\hline 6 & $\begin{array}{l}\text { Adaptabilidad: flexibilidad ante los } \\
\text { cambios. }\end{array}$ & $14,3 \%$ & $71,4 \%$ & $14,3 \%$ & $57,1 \%$ & $28,6 \%$ & $37,1 \%$ \\
\hline 7 & $\begin{array}{l}\text { Logro: impulso por satisfacer y mejorar } \\
\text { objetivos propios de la excelencia. }\end{array}$ & $28,6 \%$ & $57,1 \%$ & $14,3 \%$ & $14,3 \%$ & $57,1 \%$ & $34,3 \%$ \\
\hline 8 & $\begin{array}{l}\text { Iniciativa: disposición para actuar sobre } \\
\text { las oportunidades que se presentan }\end{array}$ & $42,8 \%$ & $42,8 \%$ & $42,8 \%$ & $28,6 \%$ & $42,8 \%$ & $40,0 \%$ \\
\hline 9 & $\begin{array}{l}\text { Optimismo: capacidad para insistir } \\
\text { en la consecución de objetivos pese a } \\
\text { obstáculos. }\end{array}$ & $14,3 \%$ & $85,7 \%$ & $28,6 \%$ & $57,1 \%$ & $57,1 \%$ & $48,5 \%$ \\
\hline \multicolumn{8}{|c|}{ Dimensión 3. Conciencia social: desde una óptica relacionada con su entorno. } \\
\hline 10 & $\begin{array}{l}\text { Empatía: capacidad para interiorizar } \\
\text { sentimientos y perspectivas de los demás } \\
\text { actuando activamente en sus preocupa- } \\
\text { ciones. }\end{array}$ & $28,6 \%$ & $71,4 \%$ & $14,3 \%$ & $71,4 \%$ & $42,8 \%$ & $45,7 \%$ \\
\hline 11 & $\begin{array}{l}\text { Conciencia organizacional: capacidad } \\
\text { para interpretar corrientes emocionales } \\
\text { de un grupo y relaciones de poder exis- } \\
\text { tentes. }\end{array}$ & $28,6 \%$ & $42,8 \%$ & $14,3 \%$ & $57,1 \%$ & $28,6 \%$ & $34,3 \%$ \\
\hline 12 & $\begin{array}{l}\text { Orientación de servicio: anticipar, reco- } \\
\text { nocer y satisfacer las necesidades de los } \\
\text { clientes. }\end{array}$ & $0,0 \%$ & $14,3 \%$ & $14,3 \%$ & $71,4 \%$ & $28,6 \%$ & $25,7 \%$ \\
\hline \multicolumn{8}{|c|}{$\begin{array}{l}\text { Dimensión } 4 \text {. Gestión de las relaciones: desde una visión emprendedora relacionada con la gestión de los } \\
\text { recursos humanos. }\end{array}$} \\
\hline 13 & $\begin{array}{l}\text { Desarrollo de personas: detección de las } \\
\text { necesidades de desarrollo de las perso- } \\
\text { nas, así como el fortalecimiento de sus } \\
\text { capacidades. }\end{array}$ & $28,6 \%$ & $42,8 \%$ & $0,0 \%$ & $57,1 \%$ & $42,8 \%$ & $34,3 \%$ \\
\hline 14 & $\begin{array}{l}\text { Liderazgo inspirador: inspirar a las } \\
\text { personas bajo un liderazgo efectivo. }\end{array}$ & $14,3 \%$ & $71,4 \%$ & $0,0 \%$ & $57,1 \%$ & $42,8 \%$ & $37,1 \%$ \\
\hline
\end{tabular}




\begin{tabular}{|c|l|c|c|c|c|c|c|}
\hline 15 & $\begin{array}{l}\text { Catalizar el cambio: iniciar o propulsar } \\
\text { la gestión del cambio. }\end{array}$ & $42,8 \%$ & $28,6 \%$ & $14,3 \%$ & $28,6 \%$ & $28,6 \%$ & $28,6 \%$ \\
\hline 16 & $\begin{array}{l}\text { Influencia: implantar actividades orien- } \\
\text { tadas a la persuasión. }\end{array}$ & $0,0 \%$ & $71,4 \%$ & $0,0 \%$ & $57,1 \%$ & $57, \%$ & $37,1 \%$ \\
\hline 17 & $\begin{array}{l}\text { Gestión de conflictos: negociación y } \\
\text { resolución de desacuerdos. }\end{array}$ & $42,8 \%$ & $57,1 \%$ & $14,3 \%$ & $42,8 \%$ & $28,6 \%$ & $37,1 \%$ \\
\hline 18 & $\begin{array}{l}\text { Trabajo en equipo y colaboración: traba- } \\
\text { jar con otros hacia metas compartidas. }\end{array}$ & $42,8 \%$ & $28,6 \%$ & $0,0 \%$ & $57,1 \%$ & $71,4 \%$ & $40,0 \%$ \\
\hline
\end{tabular}

Fuente: elaboración propia.

También las competencias de la dimensión 3, «conciencia social», servirían para dar respuesta a cuatro de los cinco escenarios planteados (con la excepción del 1, que son los aspectos institucionales y legales). En esta dimensión, tendría un especial peso la competencia identificada como «empatía» $(45,7 \%)$, un aspecto clave en los escenarios psicofísico y sociocultural.

Respecto a la dimensión 1, «autoconocimiento», contiene competencias que responderían a varios de los escenarios considerados, con la excepción del legalinstitucional y el económico, con los que — según los participantes - guarda una menor relación. En esta dimensión la «conciencia emocional» y la «autoconfianza» (ambas con un 40\%) son las destacadas por su relación con las cuestiones planteadas en los distintos escenarios.

Por último, en la dimensión 4, "gestión de las relaciones», la competencia más relevante es el «trabajo en equipo» (40\%), especialmente en el escenario educativo. Se trata de un resultado muy relevante, por lo que pueda suponer como orientación en la planificación de estrategias formativas. El resto de competencias de esta dimensión aparecen en menor medida relacionadas con la resolución de problemas planteados en los distintos escenarios; es el caso de la competencia «catalizar el cambio» (28,6\%), una de las que obtiene un menor peso porcentual - junto con la «orientación al servicio» (dimensión 3) - , de todas las competencias consideradas.

Estos resultados abren una interesante discusión en torno a los aspectos que resultan de especial relevancia en el emprendimiento de las personas con discapacidad. En este sentido, los hallazgos confirman otras investigaciones precedentes sobre el tema (Olaz y Ortiz, 2016a; 2016b; Ortiz y Olaz, 2016a; 2016b) que hallan conexiones entre las iniciativas emprendedoras (en el caso del colectivo de mujeres) y competencias individuales y sociales, como las relacionadas con el autoconocimiento, la autoevaluación, la conciencia emocional, la capacidad para gestionar los conflictos o el trabajo en equipo. 


\section{Conclusiones}

Ante la extensa relación de cuestiones que suponen un límite para el emprendimiento de las personas con discapacidad, se impone la toma de medidas que contribuyan a paliar, si no a eliminar, los obstáculos que deben superar.

Las propuestas recogidas tras la realización de la TGN revelan un conjunto de acciones que suponen un punto de partida sobre el que comenzar a trabajar.

Las que figuran a continuación son el resultado de la reflexión que los participantes en la TGN y los propios investigadores han realizado sobre el tema:

- En lo referente a los aspectos institucionales-legales, se proponen varias soluciones ligadas a las autoridades administrativas de manera que estas impulsen modificaciones en diversas áreas, tales como el sistema educativo, necesitado de una mayor adaptación a una formación específica que ahora solo facilita la empresa privada con costes elevados. Junto con la Administración, se proponen una serie de medidas relacionadas con la necesaria adaptación de las herramientas financieras a las necesidades especiales de las personas con discapacidad. En este sentido, la ampliación de la obra social de dichas entidades o la mejor adecuación de las líneas financieras existentes a las demandas de colectivos específicos, como es este, serían de gran ayuda para promover una actividad dotada de alto grado de autonomía, como es el emprendimiento.

- De forma más específica, la orientación de los recursos al aprovisionamiento de medios humanos y materiales (esencialmente tecnológicos) es una medida altamente demandada.

- Desde un punto de vista competencial, las dimensiones relacionadas con la autogestión y la gestión de relaciones guardan una alta correspondencia con las soluciones planteadas.

- En cuanto a los personales-psicológicos, las propuestas se orientan en dos direcciones. La primera pone el foco de atención en la necesidad de disponer de los medios necesarios para facilitar el aprendizaje, ya se trate de humanos (especialmente personal de apoyo, como intérpretes de lengua de signos para los usuarios de esta lengua) como de técnicos.

- La segunda dirección es la que apunta a la necesidad de disponer de recursos psicológicos que ayuden a trabajar aspectos como la autoconfianza. Una competencia que, sin duda, tiene un alto valor para cualquier acción emprendedora. 
- Una muestra de la relevancia del factor psicofísico es que, según los participantes en la TGN, todas las competencias serían necesarias a la hora de eliminar o minimizar los obstáculos para emprender.

- En tercer lugar, las soluciones aportadas a los aspectos socioeconómicos se centran en facilitar instrumentos que impulsen la actividad empresarial, como son las líneas de crédito blando, financiación a fondo perdido y otras medidas de carácter puramente económico. No obstante, la solución no pasa por la mera disponibilidad de fondos, sino por un estudio específico sobre las necesidades financieras adaptadas a este colectivo.

- Junto con las anteriores, algunas ideas son especialmente relevantes. En este sentido, se propone la creación de centros de trabajo para emprendedores discapacitados. Desde una perspectiva competencial, este conjunto de factores estaría especialmente relacionado con las dimensiones de «autogestión»y «conciencia social».

- Las soluciones indicadas para los aspectos socioculturales tienen que ver fundamentalmente con la sensibilización social hacia las particularidades de las personas con discapacidad. En este sentido sería necesario insistir en la importante labor que las Administraciones deben realizar para visibilizar no ya al discapacitado, sino a todas sus potencialidades profesionales y personales.

- La normalización de la discapacidad pasa por la inserción de estas personas en todos los ámbitos sociales y, para ello, desde la Administración educativa hasta la de ámbito económico deben estar involucradas. Es cierto que se ha avanzado en la sensibilización social hacia unas personas «diferentes», pero, a la vista de las reivindicaciones realizadas, queda claro que hay mucho por hacer, de la escuela a la empresa y desde la «cuna» hasta la «tumba».

- En este escenario aparecen relacionadas todas las competencias. Ello muestra el esfuerzo a realizar por parte de la persona, de una forma integral, para vencer las dificultades que previamente se han enunciado.

- Por último, en el aspecto educativo-formativo, las soluciones pasan por ofertar formación, pero no cualquier tipo de formación, sino aquella muy específicamente diseñada y adaptada atendiendo a las necesidades concretas del colectivo.

- La accesibilidad a los recursos de forma sencilla y con asistencia personalizada es un requisito para que el esfuerzo adicional que deben hacer 
las personas discapacitadas a la hora de la formación no sea un elemento disuasorio.

- La eliminación de los obstáculos relacionados con la formación y su canalización en una actividad emprendedora implica el despliegue de todas las competencias psicológicas y sociales de la persona discapacitada.

Los hallazgos de esta investigación constituyen un punto de partida a partir del cual se debe seguir profundizando en aspectos sobre los que este estudio puede arrojar luz.

Resulta de especial interés estudiar la conexión entre competencias y factores determinantes del emprendimiento, si bien, en este caso, partiendo de una selección de aquellas que han mostrado una mayor capacidad explicativa sobre la actitud emprendedora en personas con discapacidad, que ha sido la principal aportación de este trabajo.

\section{Referencias bibliográficas}

AJzen, Icek (1987). "Attitudes, traits and actions: dispositional prediction of behaviour in social psychology." Advances in Experimental Social Psychology, 20, 1-63.

Ajzen, Icek (1988). Attitudes, Personality and Behaviour. Chicago: Dorsey.

Bascones, Luis Miguel y Quezada, Martha Yolanda (2006). Daño cerebral sobrevenido en Castilla-La Mancha: realidad sociosanitaria, desafíos de apoyo e integración comunitaria. Toledo: FISLEM.

Benavides, María del Mar, Sánchez, María Isabel y Luna, Roberto (2004). "El proceso de aprendizaje para los emprendedores en la situación actual: un análisis cualitativo en el ámbito universitario". Revista de dirección, organización y administración de empresas, 30, 34-48.

Bonnett, Celia y Furnham, Adrian (1991)."Who wants to be an entrepreneur? A study of adolescents interested in a young enterprise scheme". Journal of Economic Psychology, 12, 465-478.

Brockhaus, Robert (1980). "Risk taking propensity of entrepreneurs." Academy of Management Journal, 23, 63-77.

Casado, Demetrio (1991). Panorámica de la discapacidad. Barcelona: Ed. Intress.

Cromie, Stanley (2000)."Assesing entrepreneurial inclinations: some approaches and empirical evidence". European Journal of Work and Organizational Psychology, 9 (1), 7-30.

De Lorenzo, Rafael (2003). El futuro de las personas con discapacidad en el mundo. Madrid: Fundación ONCE. 
Delbece, André y Van de Ven, Andrew (1971). "A Group Process Model for Problem Identification and Program Planning". Journal of Applied Behavioral Science, 7 (4), 466-492

Delbecq, André, Van de Ven, Andrew y Gustafson, David (1975). Group techniques for program planning: A guide to nominal group and Delphi processes. Glenview, IL: Scott, Foresman.

DíAz, Máximo (1985). El asociacionismo de los minusválidos entre organización y movimiento social. Madrid: Universidad Complutense de Madrid.

Dobbie, Allison, Rhodes, Marthin, Tysinger, James y Freeman, Joshua (2004). "Using a Modified Nominal Group Technique as a Curriculum Evaluation Tool". Family Medicine, 36 (6), 402-406.

Evans, David y Leighton, Linda (1989). "Some empirical aspects of entrepreneurship." American Economic Review, 79 (3).

Fernández-Cid, Matilde (2005). Representaciones de la discapacidad intelectual (tesis doctoral). Universidad Complutense de Madrid. Recuperado de: <http://cisne.sim.ucm.es/search*spi/afernandez+cid/afernandez+cid/ $1 \% 2 \mathrm{C} 2 \% 2 \mathrm{C} 2 \% 2 \mathrm{CB} /$ frameset $\& \mathrm{FF}=$ afernande $z+\mathrm{cid}+$ enrique $z+$ matilrde $\&$ $1 \% 2 \mathrm{C} 1 \% 2 \mathrm{C}>$.

Gutiérrez, Jesús (2011). “Grupo de Discusiónः ¿Prolongación, variación o ruptura con el focus group?" Cinta moebio, 41, 105-122.

HAY GROUP, McClelland Center for Research and Innovation (2005). Prepared by Steven B. Wolff, DBA. Updated November 2005. Emotional Competence Inventory (ECI) Technical Manual.

INE (Instituto Nacional de Estadística) (1986). Encuesta sobre Discapacidades, Deficiencias y Minusvalias 1986 (EDDM 1986).

INE (Instituto Nacional de Estadística) (1999). Encuesta sobre Discapacidades, Deficiencias y Estado de Salud (EDDS 1999).

INE (Instituto Nacional de Estadística) (2008). Encuesta sobre Discapacidades, Autonomía personal y situaciones de Dependencia 2008 (EDAD 2008). Recuperado de: <http://www.ine.es/jaxi/menu.do?type=pcaxis $\&$ path $=/ \mathrm{t} 15 / \mathrm{p} 418 \&$ file $=$ inebase $>$.

Jiménez, Antonio (1991). Infancia y discapacidad. Una aproximación cuantitativa. Primer Congreso Internacional Infancia y Discapacidad, vol. 2. Madrid: Ministerio de Asuntos Sociales.

Jiménez, Antonio (1992). Tipología y Prevalencia en España de las Deficiencias. Curso de Prevención de Deficiencias. Madrid: Real Patronato de Prevención y de Atención a Personas con Minusvalía.

Jiménez, Antonio (1998a). El impacto de las nuevas tecnologías en el empleo de las personas con discapacidad. La situación del empleo de las personas con dis- 
capacidad en España. Propuestas para su reactivación. Madrid: Escuela Libre Editorial.

Jiménez, Antonio (1998b). "Jóvenes con discapacidad. Características y necesidades". Juventud y Discapacidad, 43, 13-21.

Jiménez, Antonio (2001). "La imagen social de la discapacidad". Jornadas sobre discapacidad y medios de comunicación, Cuenca, Asociación Roosevelt. Recuperado deः <http //usuarios.discapnet.es/Ajimenez/imagen/imagensocial.htm>.

Jiménez, Antonio (2002). Estudio sociodemográfico de la población con discapacidad en el Principado de Asturias. Oviedo: Fundación FASAD.

Jiménez, Antonio (2005). "Bases demográficas: estimación, características y perfiles de las personas en situación de dependencia”. Libro Blanco sobre la atención a las personas en situación de dependencia en España. Madrid: Ministerio de Trabajo y Asuntos Sociales.

Jiménez, Antonio (2007)."Conceptos y tipologías de la discapacidad: documentos y normativas de clasificación más relevantes". En Rafael de Lorenzo y Luis Cayo Pérez Bueno (coords.) (2007). Tratado sobre discapacidad. Navarra: Thomson Reuters-Aranzadi.

Jiménez, Antonio y Huete, Agustín (2002a). La discapacidad en cifras. Madrid: IMSERSO.

Jiménez, Antonio y Huete, Agustín (2002b). La discriminación por motivos de discapacidad. Análisis de las respuestas recibidas al cuestionario sobre discriminación por motivos de discapacidad promovido por el CERMI Estatal. Madrid: CERMI.

Jiménez, Antonio y Huete, Agustín (2003). Las discapacidades en España: Datos estadísticos. Madrid: Real Patronato sobre Discapacidad.

Jung, Dong; Ehrlich, Sanford; De Noble, Alex y Baik, Ki (2001). "Entrepreneurial Self-Efficacy and its Relationship to Entrepreneurial Actions: A Comparative Study between the US and Korea." Management International, 6, 41-53.

Markman, Gideon y Baron, Robert (2003). "Person-entrepreneurship fit: why some people are more successful as entrepreneurs than others". Human Resource Management Review, 13, 281-301.

Moriano, Juan Antonio (2005). El perfil psicosocial del emprendedor. Madrid: CES. Moriano, J. A., Palací, Francisco y Morales, José (2006). “Adaptación y validación en España de la escala de Autoeficacia Emprendedora". Revista de Psicología Social, 21 (1), 51-64.

Muñoz, David y Santos, Antonio (2016). Emprendedores a la fuerza: la expansión idea de capital bumano en los discursos de los jóvenes universitarios españoles. Madrid: XII Congreso Español de Sociología. 
Olaz, Ángel y Ortiz, Pilar (2016a). "The competencial factor like an engine venture". Suma de Negocios, 7 (15), 2-8.

Olaz, Ángel y Ortiz, Pilar (2016b). Competencias y emprendimiento por razón de género. Notas metodológicas a un proyecto de investigación. Madrid: Aranzadi.

OMS (2001). Clasificación Internacional del Funcionamiento, de la Discapacidad y de la Salud. Madrid: Ministerio de Trabajo y Asuntos Sociales. Secretaría General de Asuntos Sociales. Instituto de Migraciones y Servicios Sociales (IMSERSO).

Ortiz, Pilar (2016). "Actitud emprendedora de los jóvenes españoles en un contexto de crisis". Revista de Ciencias Sociales, XXII (1), 139-152.

Ortiz, Pilar y Millán, Ana (2010). "El perfil social del emprendedor." En Antonio Aragón y Samuel Baixauli (eds.). El reto de emprender: factores clave. Navarra: Thomson Reuters.

Ortiz, Pilar y Olaz, Ángel (2016a)."Elements that contribute to boost female entrepreneurship: A prospective analysis". Suma de Negocios, 7 (15), 54-60.

Ortiz, Pilar y Olaz, Ángel (2016b). Mujer y emprendimiento desde una perspectiva competencial. Madrid: Aranzadi.

Pérez, Luis (2004). El desmantelamiento de la discapacidad y otros escritos vacilantes. Barcelona: El Cobre.

Renau, Juan José (1994). Creación de empresas. De la idea al proyecto empresarial. Valencia: IMPIVA.

Renko, Maija; Parker Harris, Sarah y Caldwell, Kate (2016). "Entrepreneurial entry by people with disabilities." International Small Business Journal, 34 (5), 555-578.

Rodríguez, Gregorio (2002). Apuntes sobre el Estado del Bienestar. Madrid: Universidad de Alcalá de Henares.

Rodríguez, Gregorio y Pérez, Manuel (2004). Pobreza y exclusión social en el Principado de Asturias. Madrid: Consejo Superior de Investigaciones Científicas.

RomañaCh, Javier (2002). Héroes y parias: la dignidad en la discapacidad. Recuperado de: <http //www.minusval2000.com/relaciones/vidaIndependiente/ heroes_y_parias,html $>$.

Romañach, Javier y Palacios, Agustina (2007). El modelo de la diversidad. La Bioética y los Derechos Humanos como herramientas para alcanzar la plena dignidad en la diversidad funcional. Valencia: Generalitat Valenciana ENIL.

Ruiz, Jorge (2012). "El grupo triangular: reflexiones metodológicas en torno a dos experiencias de investigación". Empiria. Revista de Metodología de Ciencias Sociales, 24, 141-162. 
Sánchez Almagro, María Luisa (2003). Perfil psicológico del autoempleado (tesis doctoral). Universidad Complutense de Madrid.

Santos, Antonio (2014). "La política en manos de los empresarios: el imparable ascenso de la ideología del emprendedor". Papeles de relaciones Ecosociales y cambio global, 227, 29-43.

Schumpeter, Joseph (1984). Capitalismo, socialismo y democracia. Barcelona: Folio.

Shapero, Albert y Sokol, Lisa (1982). The social dimensions of entreprenership. Encyclopedia of entreprenership. New Jersey: Prentice Hall.

Suárez, Borja (2017). "Autoempleo (y emprendimiento) juvenil: ‘ahuyentar a los jóvenes de los derechos y garantías laborales?/Youth self-employment (and entrepreneurship): chasing away young workers from labor rights and guarantees?" Cuadernos de Relaciones Laborales, 35 (1), 151.

Turró, Andreu, Urbano, David y Peris-Ortiz, Marta (2014). "Culture and innovation: The moderating effect of cultural values on corporate entrepreneurship". Technological Forecasting and Social Change, 88, 360-369.

Vázquez Ferreira, Miguel Ángel (2005). "La reflexividad social transductiva: la construcción práctico-cognitiva de lo social y la sociología”. Nómadas. Revista Crítica de Ciencias Sociales y Jurídicas, 11.

Vázquez Ferreira, Miguel Ángel (2007a). Prácticas sociales, identidad y estratificación: tres vértices de un hecho social, la discapacidad. Intersticios. Revista Sociológica de Pensamiento Crítico, 1 (2), 1-14,

Vázquez Ferreira, Miguel Ángel (2007b). "Un nuevo concepto para la comprensión de la acción social: la transductividad creativa de las prácticas cotidianas”. Intersticios. Revista Sociológica de Pensamiento Crítico, 1 (1), 1-16.

VÁzquez Ferreira, Miguel Ángel (2008). “Una aproximación sociológica a la discapacidad desde el modelo social: apuntes caracteriológicos". REIS, 124, 141-174.

Vázquez Ferreira, Miguel Ángel (2010). "De la minus-valía a la diversidad funcional: un nuevo marco teórico-metodológico". Politica y Sociedad, 47 (1), 45-65.

Vázquez Ferreira, Miguel Ángel (2011). “Conformaciones, performaciones y transformaciones; herramientas transductivas de la contemporaneidad". Intersticios. Revista sociológica de pensamiento crítico, 5 (2), 5-16.

VAn de Ven, Andrew y DelbecQ, André (1972). "The nominal group as a research instrument for exploratory health studies". American journal of public bealth, 62 (3), 337-342.

Zarco, Juan y García de La Cruz, Juna José (2004). El espejo social de la mujer con gran discapacidad. Madrid: Editorial Fundamentos. 